\title{
Laser interstitial thermal therapy for the treatment of epilepsy: evidence to date
}

\author{
This article was published in the following Dove Press journal: \\ Neuropsychiatric Disease and Treatment \\ 26 September 2017 \\ Number of times this article has been viewed
}

\section{Navika D Shukla \\ Allen L Ho \\ Arjun V Pendharkar \\ Eric S Sussman \\ Casey $\mathrm{H}$ Halpern}

Department of Neurosurgery, Stanford University, Stanford, CA, USA
Correspondence: Casey H Halpern Department of Neurosurgery, Stanford University, 300 Pasteur Dr A30I, MC

5325, Stanford, CA 94305, USA

$\mathrm{Tel}+$ I 6507235574

Fax + I 6507250390

Email chalpern@stanford.edu

\begin{abstract}
Medically intractable epilepsy is associated with increased morbidity and mortality. For those with focal epilepsy and correlated electrophysiological or radiographic features, open surgical resection can achieve high rates of seizure control, but can be associated with neurologic deficits and cognitive effects. Recent innovations have allowed for more minimally invasive methods of surgical seizure control such as magnetic resonance-guided laser interstitial therapy (MRgLITT). MRgLITT achieves the goal of ablating seizure foci while preserving neuropsychological function and offering real-time feedback and monitoring of tissue ablation. This review summarizes the utilization of MRgLITT for mesial temporal lobe epilepsy and other seizure disorders. Overall, the efficacy of MRgLITT is comparable to that of open surgery and offers a less invasive approach in patients with significantly less morbidity.
\end{abstract}

Keywords: laser ablation, MRgLITT, mesial temporal lobe epilepsy, epilepsy surgery, corpus callostomy

\section{Introduction}

Of the $1 \%$ of the US population with epilepsy, one-third suffer from medically intractable epilepsy, defined as continued seizures despite the use of two separate drug regimens. ${ }^{1}$ In order to minimize the morbidity and mortality in these patients, surgical treatment options must be considered. Patients with focal epilepsy are generally good candidates for seizure control through surgery, which has demonstrated high efficacy in achieving seizure freedom. ${ }^{2}$ The gold standard for surgical control is open surgical resections aimed at localizing and excising the epileptogenic zone to achieve seizure freedom. In temporal lobe epilepsy, the most common form of drug-resistant epilepsy, open surgery involves anterior temporal lobectomy (ATL) or, as an alternative option, selective amygdalohippocampotomy (SAH), which spares resection of the anterior temporal lobe. ${ }^{3}$ Despite demonstrating satisfactory rates of seizure freedom, concern around the impact of open surgery on neuropsychological function has driven interest in minimally invasive techniques for surgical epilepsy treatment.

As an alternative to open surgery, magnetic resonance-guided laser interstitial thermal therapy (MRgLITT) is a minimally invasive option for ablation of epileptogenic foci. MRgLITT is generally offered as first-line treatment over open surgery in patients with dominant mesial temporal sclerosis (MTS) or with epileptogenic foci near deep, eloquently situated brain structures. ${ }^{1}$ In cases where the patient presents with multiple comorbidities or there are other factors that suggest open surgery may not be tolerated, MRgLITT is also usually a good option. The indications for MRgLITT are summarized in Table 1. 
Table I Indications for MRgLITT in patients with epilepsy

\section{Indications}

Mesial temporal sclerosis

Tuberous sclerosis

Cortical dysplasia

Hypothalamic hamartoma

Periventricular nodular dysplasia

Other neoplasms

Radiation necrosis

Abbreviation: MRgLITT, magnetic resonance-guided laser interstitial therapy.

Compared to other minimally invasive techniques, particularly radiosurgery, MRgLITT provides an extremely well-demarcated region of ablation and is not associated with long-term radiation risks. ${ }^{1,4}$ MRgLITT offers thermal destruction of benign or malignant tissue and has been utilized in many diseases including tumors, epilepsy, and chronic pain. The procedure has been studied in the ablation of deep, eloquently situated brain tumors, epileptogenic foci, and cingulum. Thermal destruction of target tissue occurs via the delivery of low-voltage laser energy via an optical fiber. Laser energy is converted to thermal energy, activating a cascade of enzyme induction, protein denaturation, membrane dissolution, and vessel sclerosis, ultimately leading to coagulative necrosis. ${ }^{5}$ It is important to note that once ablated, tissue cannot be obtained for pathology; however, the zone of thermal damage can be monitored in real-time via magnetic resonance imaging (MRI) with automated feedback. Placement of the laser probe within the intracranial space relies on varying methods of stereotaxy, but the use of real-time MRI stereotaxy has been shown to improve operative efficiency. ${ }^{6}$ With decreased rates of perioperative and surgical morbidity, immediate radiographic visualization of the area being treated, and a less invasive target ablation, it is hoped that MRgLITT may increase the utilization of surgical therapies for medically intractable epilepsy.

\section{Procedure}

The procedure for MRgLITT for epilepsy involves selective thermal ablation of epileptogenic foci. Depending on the method of stereotaxy chosen for the placement of the fiber-optic applicator, ablation can occur in either one or two phases. Most commonly, placement of the fiber-optic applicator occurs under stereotactic guidance within the operating room (OR) and laser ablation occurs within the MRI suite. ${ }^{7,8}$ Prior to entering the OR, skull fiducial placement and computed tomography acquisition are completed. Once within the OR, general anesthesia is administered and the patient's head is fixed with MRI-compatible Mayfield pins with the patient in the prone position. A small incision and twist drill burr hole are required before the LITT laser can be positioned in the planned target tissue. After laser placement is complete with standard stereotaxy, patients are transported to an MRI suite for laser ablation. Alternatively, with recent advances, it is possible to perform the entire procedure within the MRI suite using a realtime MRI system, such as the ClearPoint platform, for neuronavigation. ${ }^{6}$ The ClearPoint platform consists of a MRI-compatible percutaneous SMART-frame, a hand-held twist drill, ceramic stylet, and a trajectory guide tower. Use of the platform obviates the need for skull fiducial markers or a stereotactic computed tomography scan. Instead of being administered in the OR, general anesthesia is induced in the antechamber of the MRI suite. Once in the MRI suite, patient positioning, head fixation, determination of the laser's trajectory, and creation of a twist drill burr hole can all be accomplished via the ClearPoint platform. An MRI scan is obtained to confirm laser placement before moving on to ablation.

The procedure for ablation remains standardized irrespective of the method of stereotaxy utilized. Of the two commercially available MRgLITT systems in current use, the Visualase ${ }^{\circledR}$ thermal therapy (Medtronic, MN, USA) and NeuroBlate ${ }^{\circledR}$ Systems (Monteris Medical, Minneapolis, MN, USA), Visualase is most often utilized in the case of epilepsy. ${ }^{5}$ The Visualase system consists of a saline-cooled fiber-optic laser applicator with a $15 \mathrm{~W}, 980 \mathrm{~nm}$ diode laser inserted into the target epileptogenic foci. ${ }^{5}$ The trajectory of the laser is chosen in a way to ensure full ablation of the target structure(s) with minimal thermal injury to nearby structures and vasculature. In the case of mesial temporal lobe epilepsy (MTLE), an occipital trajectory is used to access the amygdalohippocampal structures. ${ }^{9}$ The aim is full ablation of mesial structures from the anterior amygdala to the posterior hippocampus at the level of the tectum.

\section{MTLE}

MTLE, the most common form of drug-resistant epilepsy, most commonly presents with MTS. Patients presenting with MTS along with electrophysiological features correlated with focal cortical lesions are generally good candidates for an ATL or a selective amygdalohippocampotomy, achieving seizure freedom rates ranging from $60 \%$ to $80 \% .^{9}$ These open surgical procedures, however, are associated with cognitive impairments and possible neurological deficits. Real-time magnetic resonance-guided stereotactic laser amygdalohippocampotomy (SLAH) is an alternative to 
ATL and SAH that could minimize these adverse events (Figure 1).

An early study by Willie et $\mathrm{al}^{9}$ reports on the use of SLAH on 13 adult patients with MTLE, of whom 9 presented with MTS. 54\% of all patients reached seizure freedom, with $67 \%$ of those with MTS achieving seizure freedom. A meaningful reduction in seizure frequency was demonstrated in $77 \%$ of patients. Follow-up ranged from 5 to 26 months, and the median length of hospitalization was one day. There was one case of a visual field defect resulting from deviated insertion of a stereotactic aligning rod. Kang et $\mathrm{al}^{10}$ reported similar results in a study of 20 patients with MTLE who were treated with MRgLITT. At 6 months postablation, 8 of 15 or $53 \%$ were seizure free. At 1 year, this number was $36.4 \%$ (4 of 11 patients), and at 2 years it was $60 \%$ (3 of 5 patients).
Four patients from this cohort went on to have ATL, and three of these patients achieved seizure freedom.

Waseem et al ${ }^{11}$ compared SLAH to SAH in patients above the age of 50 with MTLE. $80 \%$ (4 of 5) of patients receiving therapy via $\mathrm{MRgLITT}$ achieved seizure freedom compared to $100 \%$ (7 of 7) of those who received SAH. ${ }^{11}$ Mean follow-up was 1 year for the MRgLITT group and 1.8 years for the SAH group. Neuropsychological outcomes were comparable between the two groups. MRgLITT reduced the median length of hospitalization and the need for pain medication postsurgery. A more recent study by Waseem et $\mathrm{al}^{12}$ looked at a larger cohort of 38 patients with MTLE undergoing MRgLITT, 34 of whom had follow-up data. 53\% of patients demonstrated seizure freedom, and 12 postprocedural complications were noted. There were seven visual field
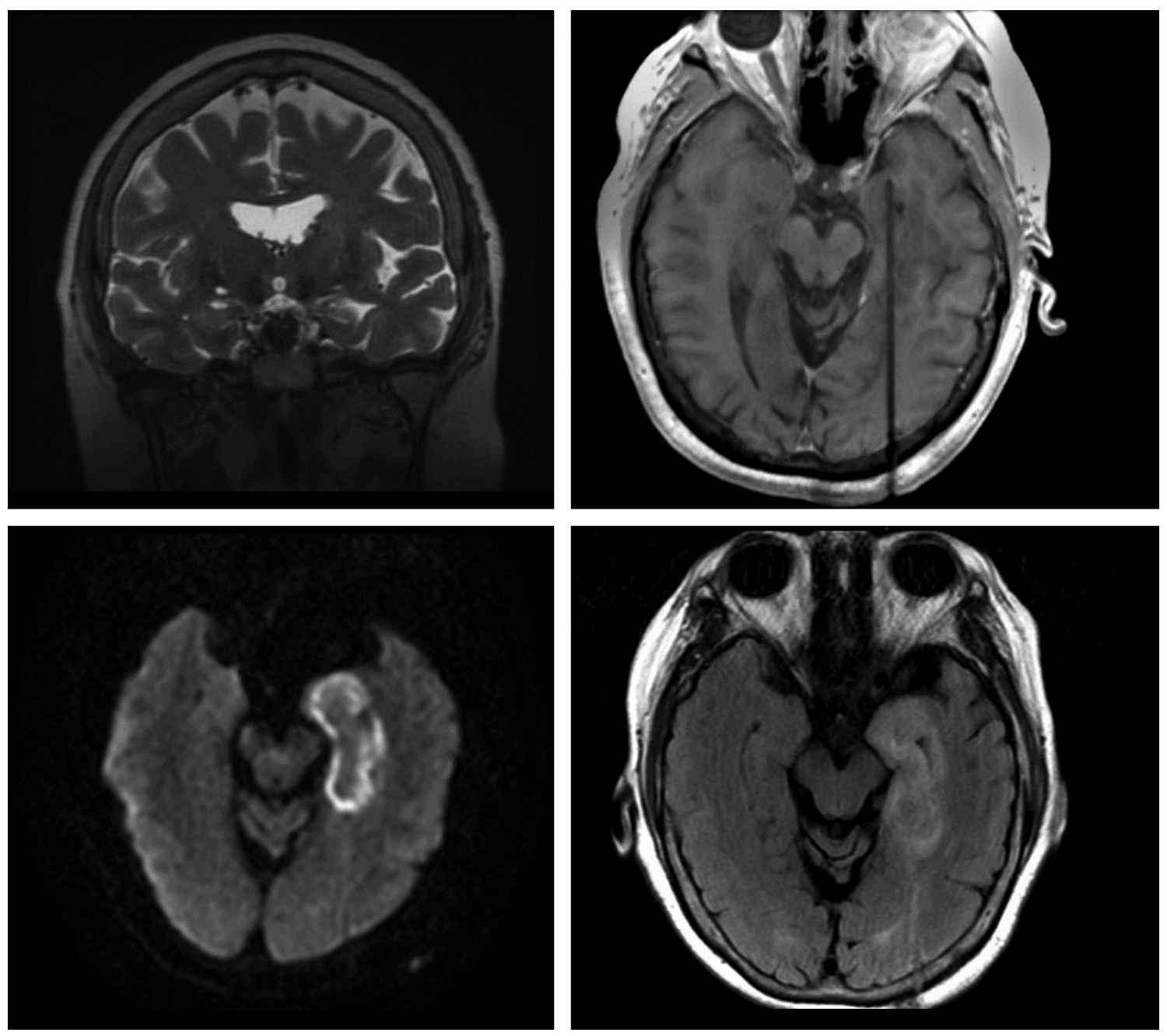

Figure I Real-time MR-guided SLAH.

Notes: (Top left) Preoperative T2-weighted coronal MRI demonstrating left sided MTS. (Top right) Intraoperative TI-weighted oblique MRI demonstrating placement of the laser fiber along the mesial temporal structures. (Bottom left) Intraoperative DWI axial MRI demonstrating extent of laser ablation along mesial temporal structures. (Bottom right) Postoperative T2 FLAIR axial MRI demonstrating area of ablation along mesial temporal structures.

Abbreviations: DWI, diffusion-weighted imaging; FLAIR, fluid-attenuated inversion recovery; MR, magnetic resonance; MRI, magnetic resonance imaging; MTS, mesial temporal sclerosis; SLAH, stereotactic laser amygdalohippocampotomy. 
cuts, three cases of intracranial hemorrhage, and two cases of cranial nerve deficits. The authors attributed the visual field cuts to issues with laser fiber placement and unintended laser heat spread along ventricular spaces. Given this, it is important, within the target structures, to avoid spread of ablation to Meyer's loop optic radiation, and to especially limit heat exposure to surrounding anatomy along the posterior hippocampus. A subset of 28 patients with MTLE also presented with MTS. Of this group, 60.7\% achieved seizure freedom. Decreased length of hospitalization, procedure time, and analgesic requirement were associated with MRgLITT compared to open surgery. Table 2 summarizes the outcome data for MRgLITT with MTLE. Seizure freedom rates are slightly higher in cases of MTLE with evidence of MTS.

Emerging technologies have enabled increased operative efficiency for MRgLITT for SLAH. Ho et $\mathrm{al}^{6}$ reported on utilizing a real-time MRI platform for completion of the entire procedure in the MRI suite. Rapid adoption of this new platform for MRgLITT was intuitive and led to decreased case times across the board, with preservation of accuracy and minimal learning curve. In terms of effects on cognitive function, significant deficits in category-related object recognition, naming, verbal learning, and memory performance are commonly seen after open resection procedures in the temporal lobe for epilepsy. Several studies have demonstrated the preservation of these functions following MRgLITT. Drane et $\mathrm{al}^{13}$ compared object naming and recognition tests between 19 patients undergoing MRgLITT for SLAH compared to a comparison cohort of 39 patients undergoing ATL and SAH in a prospective fashion and demonstrated that MRgLITT better preserves object recognition and naming. Similarly, Kang et al ${ }^{10}$ report that MRgLITT better preserves verbal learning and memory performance compared to open resection. Within their cohort of 15 patients, contextual verbal memory performance was preserved, but a decline in noncontextual memory tasks was noted. Given this data, while rates of seizure freedom with MRgLITT are slightly lower than with open surgery,

Table 2 Seizure freedom rates after MRgLITT in patients with MTLE independent of MTS status

\begin{tabular}{lll}
\hline $\begin{array}{l}\text { MRgLITT for } \\
\text { MTLE }\end{array}$ & $\begin{array}{l}\text { Sample } \\
\text { size }\end{array}$ & $\begin{array}{l}\text { Seizure freedom } \\
\text { rate }\end{array}$ \\
\hline Willie et al, ${ }^{9} 2014$ & 13 & $54 \%$ \\
Kang et al, ${ }^{10} 2016$ & 15 & $53 \%$ \\
Waseem et al, ${ }^{\prime \prime} 2015$ & 5 & $80 \%$ \\
Waseem et al, ${ }^{12} 2017$ & 34 & $53 \%$ \\
\hline
\end{tabular}

Abbreviations: MRgLITT, magnetic resonance-guided laser interstitial therapy; MTLE, mesial temporal lobe epilepsy; MTS, mesial temporal sclerosis.
MRgLITT may be especially valuable for high-risk patients or patients steered away by the risks of open surgery. In cases where MRgLITT is unsuccessful, the capacity to perform a second MRgLITT or open surgery is preserved.

\section{Cortical ablations}

MRgLITT has been most extensively studied in the context of MTLE, but has also demonstrated efficacy in the treatment of epileptogenic foci other than those characterizing MTLE. Laser ablation for periventricular nodular heterotopia (PVH), a condition characterized by improper neuronal migration, is thought to be particularly useful given the difficulty of surgically accessing PVHs. ${ }^{1}$ When functional tissue prevents open access to the epileptogenic loci, laser ablation provides a method of focal destruction. Real-time temperature monitoring of surrounding tissue prevents damage to tissue outside the target lesion.

Esquenazi et $\mathrm{al}^{14}$ reported the first use of MRgLITT for PVHs on two patients, a 48-year-old female and a 25 -year-old male. Both patients tolerated the procedure well and were discharged on postoperative day one. Patient two (male) developed a complete right homonymous hemianopsia, which responded to treatment with steroids. Patient one was seizure free for one month postablation after which seizures recurred. Medication adjustment resulted in seizure freedom at 12 months for this patient. Patient two remained seizure free for 2 months after which seizures recurred. Seizure freedom was achieved 9 months after an ATL and amygdalohippocampotomy. A third patient, discussed by Clarke et $\mathrm{a}^{15}$ received MRgLITT for the treatment of bilateral occipital dysplasia with PVHs extending from the periventricular region to the occipital lobe, surrounded by eloquent cortex. The proximity of eloquent cortex to the lesion ruled out the use of open resection. After MRgLITT, the patient remained seizure free at 8 months follow-up with no neurological deficits. More recently, Thompson et $\mathrm{al}^{16}$ reported on the use of MRgLITT in two case studies of PVH. One patient, a 28-year-old male with medically refractory seizures of complex origin involving PVHs and mesial temporal structures, was treated with both a right anterior temporal lobectomy and interval ablation of the remaining right hemispheric nodules by MRgLITT. At one year postablation, the patient remained seizure free. The second patient, a 32-year-old female, was treated with MRgLITT for medically refractory epilepsy with PVH. She remained seizure free at 6 months postsurgery. With both patients, intracranial electroencephalogram evaluation was utilized prior to surgery to adequately assess the epileptogenic network. MRgLITT was 
effective in the treatment of multifocal, complex epilepsy when coupled with intracranial EEG evaluation. Further discussion of MRgLITT for cortical ablations other than PVH is discussed in the "Pediatrics" section.

\section{Disconnection surgeries}

Unlike resective surgeries which aim to provide seizure freedom, disconnection surgeries are aimed at reducing morbidity by limiting seizure spread. Of the various disconnection procedures in use today, in this review, we will focus on laser ablation for corpus callosotomies. Introduced in 1940, the corpus callosotomy involves a partial or complete separation of the corpus callosum, the major commissural connection between the two hemispheres of the brain. ${ }^{17}$ Disconnecting the two hemispheres prevents spread of local seizure activity, thereby limiting ictal loss of consciousness and drop attacks. ${ }^{18}$ As complete seizure freedom is rare with both partial and complete callosotomies, corpus callosotomies are generally indicated in patients with multifocal tonic, atonic, or myoclonic seizures. ${ }^{18-20}$ The relative efficacy of a partial versus a complete callosotomy is still debated in the literature. ${ }^{21}$ Both have been shown to reduce seizure frequency in patients; however, partial callosotomies are thought limit the incidence of disconnection syndromes by preserving perceptual connections between hemispheres. In cases where a partial callosotomy is unsuccessful, the literature is supportive of a second procedure to complete the callosotomy. ${ }^{22}$ As a minimally invasive alternative to open surgery, MRI-guided laser interstitial thermal ablation may be particularly well-suited to performing a full or partial callosotomy. MRgLITT is most effective when used to ablate relatively homogenous tissues with uniform absorbance properties. $^{7,23}$ The homogenous nature of the corpus callosum and the capacity of the surrounding structures to act as heat sinks allows for effective focal ablation without damage to adjacent structures. ${ }^{7}$

Ho et $\mathrm{al}^{7}$ report on the efficacy of MRgLITT of the splenium to achieve a complete callosotomy on a 30 -year old patient who continued to suffer from medically refractory epilepsy despite a prior anterior corpus callosotomy. At 4 months post-MRgLITT, the patient saw a 50\% drop in seizure frequency and complete freedom from atonic seizures. Thermal ablation was well-tolerated and no neurological impairment was noted. A study by Pruitt et $\mathrm{al}^{24}$ reports on the use of MRgLITT for three corpus callosotomies for bifrontal epilepsy. Of the three cases, there was one complication due to catheter malposition. The authors do not report on the outcomes of the corpus callosotomies separate from the other cases for which laser ablation was utilized, but note that a majority of patients saw an improvement in seizure frequency.

\section{Pediatrics}

Laser ablation is a particularly attractive minimally invasive option for pediatric cases with focal, medically intractable epilepsy. Pediatric epilepsy has a prevalence of 1 in every 100 children, and roughly one-third of these cases are resistant to control with medications alone..$^{25}$ In the pediatric population, early control of epileptic seizures plays a crucial role in preventing worsening cognitive outcomes, impaired functional status, and mental health disorders. Surgical resection of medically refractory epilepsy has been shown to have long-term seizure control rates in the $50 \%-70 \%$ range, but of the 100,000-500,000 patients who are candidates for surgical resection, less than $1 \%$ receive an operation. ${ }^{25,26}$ While the reasons for this treatment gap are complex, part of the issue stems from the invasiveness and risk of complications associated with the current gold standard of craniotomy followed by resection. Laser ablation for certain epileptogenic lesions, particularly those near deep or eloquent brain structures, offers a compelling alternative. MRgLITT offers the advantage of minimal invasiveness coupled with real-time feedback. Studies done thus far in children with epilepsy have shown MRgLITT to be an effective form of treatment for lesions ranging from hypothalamic hamartomas (HH) to MTS. ${ }^{25}$

One of the earliest reviews of MRgLITT for pediatric epilepsy by Curry et $\mathrm{a}^{27}$ reports the results of five pediatric cases: two HHs, a cingulate tumor with tuberous sclerosis complex (TSC), a case of MTS, and a left frontal cortical dysplasia. At the time of follow-up, which ranged from 2 to 13 months, all patients were found to be seizure free and no complications were reported. A later report by Wilfong and Curry $^{28}$ on 14 pediatric patients with $\mathrm{HHs}$ demonstrated seizure freedom at a mean of 9 months follow-up in $86 \%$ of cases. Complications were limited to one subarachnoid hemorrhage, and median length of stay was one day. Lewis et $\mathrm{al}^{29}$ published the results of MRgLITT on 17 pediatric patients. Two patients underwent treatment twice; one due to incorrect fiber placement and the second due to seizure persistence. Twelve cases of focal cortical dysplasia, four definitive cases of TSC, one $\mathrm{HH}$, one patient with MTS, one tumor, and one case of Rasmussen encephalitis were treated with MRgLITT. Some patients were diagnosed with more than one substrate. An Engel class I outcome (seizure freedom) was achieved in $41 \%$ of patients, and an Engel 
class II outcome was achieved in one additional patient. Mean follow-up was 16.1 months, average length of stay was 1.56 days, and four patients experienced complications. The patient who underwent MRgLITT twice achieved seizure freedom after the second treatment. Table 3 summarizes the range of pediatric cases for which MRgLITT has been attempted.

The report by Lewis et $\mathrm{al}^{29}$ demonstrated lower rates of efficacy for MRgLITT compared to conventional pediatric epilepsy surgery. According to the authors, this may have been due to the high rate of more complex epilepsy among their patient sample with eight of the seventeen having had at least one prior resection. MRgLITT may be most suitable for cases in which standard open craniotomies with resection are associated with high neurologic risk such as HHs.

In the case of $\mathrm{HH}$ particularly, laser ablation is rapidly being considered as first-line therapy. ${ }^{25} \mathrm{HHs}$ are a rare congenital malformation of the hypothalamus resulting in gelastic seizures. Given their deep location and association with the hypothalamus, resecting $\mathrm{HH}$ lesions via open surgery or endoscopic procedures carries significant risks. Realtime laser ablation allows for minimal brain manipulation along with real-time assessment of the area being treated. Compared to radiosurgery, the alternative minimally invasive treatment, MRgLITT, is immediately ablative with low postprocedure side effects. In contrast, the beneficial effects of radiosurgery can be delayed up to 12 months or more postsurgery, radiation necrosis is a common concern, and seizure outcomes following radiosurgery tend to be poorer. ${ }^{10,17}$

Table 3 Applications and associated outcomes of MRgLITT in the pediatric population

\begin{tabular}{|c|c|c|}
\hline Reference & Substrate & $\begin{array}{l}\text { Overall seizure } \\
\text { freedom rate }\end{array}$ \\
\hline Curry et al, ${ }^{27} 2012$ & $\begin{array}{l}\mathrm{HH}(\mathrm{n}=2) \\
\text { Cingulate tumor } \\
\text { with TSC }(\mathrm{n}=\mathrm{I}) \\
\text { MTS }(\mathrm{n}=\mathrm{I}) \\
\text { LF cortical } \\
\text { dysplasia }(\mathrm{n}=\mathrm{I})\end{array}$ & $100 \%$ \\
\hline Wilfong and Curry, ${ }^{28} 2013$ & $\mathrm{HH}(\mathrm{n}=14)$ & $86 \%$ \\
\hline Lewis et al, ${ }^{29} 2015$ & $\begin{array}{l}\text { Focal cortical } \\
\text { dysplasia }(n=I 2) \\
\text { TSC }(n=5) \\
\text { HH }(n=I) \\
\text { MTS }(n=I) \\
\text { Tumor }(n=I) \\
\text { Rasmussen } \\
\text { encephalitis }(n=I)\end{array}$ & $41 \%$ \\
\hline
\end{tabular}

Abbreviations: $\mathrm{HH}$, hypothalamic hamartoma; LF, left frontal; MRgLITT, magnetic resonance-guided laser interstitial therapy; MTS, mesial temporal sclerosis; TSC, tuberous sclerosis complex.
Laser ablation may also be a compelling alternative for multifocal causes of epilepsy such as TSC where multiple craniotomies may be required to achieve seizure freedom. ${ }^{27}$ The one patient with TSC reported on by Curry et $\mathrm{al}^{27} \mathrm{dem}$ onstrated seizure freedom at 13 months postablation. Two of the four patients with definitive TSC in the study conducted by Lewis et a ${ }^{29}$ were seizure free at the time of follow-up.

Based off studies of laser ablation for primary glial neoplasms in adults, MRgLITT for focal epilepsy stemming from low-grade glioneuronal tumors in children is under study. ${ }^{25,30,31}$ The full range of applications of MRgLITT in the context of medically refractory epilepsy is still being explored.

\section{Conclusion}

We conclude that MRI-guided laser interstitial thermal therapy is a safe and effective therapeutic option for the management of medically intractable epilepsy in the adult and pediatric populations. Of particular significance is the minimally invasive nature of MRgLITT, which enables the surgical management of patients who are not good candidates for, or are otherwise averse to, open resection. Compared to other minimally invasive procedures, MRgLITT is associated with improved outcomes and better side effect profile. While open surgical procedures have demonstrated slightly higher rates of seizure freedom, MRgLITT is associated with reduced hospitalization time, decreased postoperative pain, and improved neuropsychological function. It is important to note, however, that the studies reviewed were limited by small samples sizes and the relative novelty of the procedure. Other limitations of the currently available data include the lack of availability of long-term outcomes data and a scarcity of randomized controlled trials. Future studies may seek to address these gaps while also looking at questions around the use of the procedure for multifocal epilepsy and the relationship between time from diagnosis and MRgLITT efficacy.

\section{Disclosure}

The authors report no conflicts of interest in this work.

\section{References}

1. LaRiviere MJ, Gross RE. Stereotactic laser ablation for medically intractable epilepsy: the next generation of minimally invasive epilepsy surgery. Front Surg. 2016;3:64.

2. Wiebe S, Blume WT, Girvin JP, Eliasziw M. A randomized, controlled trial of surgery for temporal-lobe surgery. N Engl J Med. 2001; 345(5):311-318.

3. Engel J Jr, McDermott MP, Wiebe S, et al. Early surgical therapy for drugresistant temporal lobe epilepsy: a randomized trial. JAMA. 2012;307: 922-930. 
4. Barbaro NM, Quigg M, Broshek DK, et al. A multicenter, prospective pilot study of gamma knife radiosurgery for mesial temporal lobe epilepsy: seizure response, adverse events, and verbal memory. Ann Neurol. 2009;65:167-175.

5. Lagman C, Chung LK, Pelargos PE, et al. Laser neurosurgery: a systematic analysis of magnetic resonance-guided laser interstitial thermal therapies. J Clin Neurosci. 2017;36:20-26.

6. Ho A, Sussman ES, Pendharkar AV, et al. Improved operative efficiency using a real-time MRI-guided stereotactic platform for laser amygdalohippocampotomy. J Neurosurg. 2017:1-8.

7. Ho AL, Miller KJ, Cartmell S, Inoyama K, Fisher RS, Halpern CH. Stereotactic laser ablation of the splenium for intractable epilepsy. Epilepsy Behav Case Rep. 2016;5:23-26.

8. Patel NV, Jethwa PR, Barrese JC, Hargreaves EL, Danish SF. Volumetric trends associated with MRI-guided laser-induced thermal therapy (LITT) for intracranial tumors. Lasers Surg Med. 2013;45:362-369.

9. Willie JT, Laxpati NG, Drane DL, et al. Real-time magnetic resonanceguided stereotactic laser amygdalohippocampotomy for mesial temporal lobe epilepsy. Neurosurgery. 2014;74:569-585.

10. Kang JY, Wu C, Tracy J, et al. Laser interstitial thermal therapy for medically intractable mesial temporal lobe epilepsy. Epilepsia. 2016; 57:325-334.

11. Waseem H, Osborn KE, Schoenberg MR, et al. Laser ablation therapy: an alternative treatment for medically resistant mesial temporal lobe epilepsy after age 50. Epilepsy Behav. 2015;51:152-157.

12. Waseem H, Vivas AC, Vale FL. MRI-guided laser interstitial thermal therapy for treatment of medically refractory non-lesional mesial temporal lobe epilepsy: outcomes, complications, and current limitations a review. J Clin Neurosci. 2017;38:1-7.

13. Drane DL, Loring DW, Voets NL, et al. Better object recognition and naming outcome with MRI-guided stereotactic laser amygdalohippocampotomy for temporal lobe epilepsy. Epilepsia. 2015;56: 101-113.

14. Esquenazi Y, Kalamangalam GP, Slater JD, et al. Stereotactic laser ablation of epileptogenic periventricular nodular heterotopia. Epilepsy Res. 2014;108:547-554.

15. Clarke DF, Tindall K, Lee M, Patel B. Bilateral occipital dysplasia, seizure identification, and ablation: a novel surgical technique. Epileptic Disord. 2014;16:238-243.

16. Thompson SA, Kalamangalam GP, Tandon N. Intracranial evaluation and laser ablation for epilepsy with periventricular nodular heterotopia. Seizure. 2016;41:211-216.
17. Englot DJ, Birk H, Chang EF. Seizure outcomes in nonresective epilepsy surgery: an update. Neurosurg Rev. 2017;40(2):181-194.

18. Park MS, Nakagawa E, Schoenberg MR, Benbadis SR, Vale FL. Outcome of corpus callosotomy in adults. Epilepsy Behav. 2013;28: $181-184$.

19. Spencer SS, Spencer DD, Williamson PD, Sass K, Novelly RA, Mattson RH. Corpus callosotomy for epilepsy: I. Seizure effects. Neurology. 1988;38:19.

20. Asadi-Pooya AA, Sharan A, Nei M, Sperling MR. Corpus callosotomy. Epilepsy Behav. 2008;13:271-278.

21. Malmgren K, Rydenhag B, Hallbook T. Reappraisal of corpus callosotomy. Curr Opin Neurol. 2015;28:175-181.

22. Reutens DC, Bye AM, Hopkins IJ, et al. Corpus callosotomy for intractable epilepsy: seizure outcome and prognostic factors. Epilepsia. 1993;34:904-909.

23. Quigg M, Barbaro NM. Stereotactic radiosurgery for treatment of epilepsy. Arch Neurol. 2008;65(2):177-183.

24. Pruitt R, Gamble A, Black K, Schulder M, Mehta AD. Complication avoidance in laser interstitial thermal therapy: lessons learned. J Neurosurg. 2017;126(4):1238-1245.

25. Buckley R, Estronza-Ojeda S, Ojemann JG. Laser ablation in pediatric epilepsy. Neurosurg Clin N Am. 2016;27(1):69-78.

26. Ravindra VM, Sweney MT, Bollo RJ. Recent developments in the surgical management of paediatric epilepsy. Arch Dis Child. 2017;102: 760-766.

27. Curry DJ, Gowda A, McNichols RJ, Wilfong AA. MR-guided stereotactic laser ablation of epileptogenic foci in children. Epilepsy Behav. 2012;24(4):408-414.

28. Wilfong AA, Curry DJ. Hypothalamic hamartomas: optimal approach to clinical evaluation and diagnosis. Epilepsia. 2013;54(9):109-114.

29. Lewis EC, Weil AG, Duchowny M, Bhatia S, Ragheb J, Miller I. MRguided laser interstitial thermal therapy for pediatric drug-resistant lesional epilepsy. Epilepsia. 2015;56:1590-1598.

30. Mohammadi AM, Hawasli AH, Rodriguez A, et al. The role of laser interstitial thermal therapy in enhancing progression-free survival of difficult-to-access high-grade gliomas: a multicenter study. Cancer Med. 2014;3(4):971-979

31. Sloan AE, Ahluwalia MS, Valerio-Pascua J, et al. Results of the NeuroBlate system first-in-humans Phase I clinical trial for recurren glioblastoma: clinical article. J Neurosurg. 2013;118(6):1202-1219.
Neuropsychiatric Disease and Treatment

\section{Publish your work in this journal}

Neuropsychiatric Disease and Treatment is an international, peerreviewed journal of clinical therapeutics and pharmacology focusing on concise rapid reporting of clinical or pre-clinical studies on a range of neuropsychiatric and neurological disorders. This journa is indexed on PubMed Central, the 'PsycINFO' database and CAS,

\section{Dovepress}

and is the official journal of The International Neuropsychiatric Association (INA). The manuscript management system is completely online and includes a very quick and fair peer-review system, which is all easy to use. Visit http://www.dovepress.com/testimonials.php to read real quotes from published authors. 